\title{
The effects of individual differences in ability to image on recall of nonmeaningful information
}

\author{
ROBERT G. KRAFT and JOHN A. GLOVER \\ University of Nebraska, Lincoln, Nebraska 68588
}

\begin{abstract}
A preliminary study examined the effects of imagery on the recall of nonmeaningful information. Experiment 1 found no facilitative effects, but the results suggested the possibility that individual differences in visual imagery ability might be an important variable. Experiment 2 examined individual differences in visual imagery ability and found this variable to be a significant factor in the effects that instructions to employ imagery have on recall of nonmeaningful information. Experiment 3 extended the results of Experiment 2 to an incidental learning task.
\end{abstract}

In a recent study, Ghatala (1981) investigated the effects of incidental semantic and acoustic processing of words (equated on meaningfulness) on subsequent recall. Her findings indicated that the semantic task resulted in a significantly greater rate of recall than did the acoustic task. Ghatala further determined that for words of high and low meaningfulness equated on the basis of imagery value, the semantic task resulted in a greater rate of recall only for high-meaningfulness words. Ghatala (1981, p. 47) suggested that her results indicated "that although semantic and nonsemantic tasks differentially activate the associative meaning of words, the amount of associative processing that occurs depends also on the structural characteristics of the stimulus material and the nature of the semantic task."

Ghatala's conclusions draw heavily on the research on orienting tasks (e.g., Hyde \& Jenkins, 1969, 1973; Walsh \& Jenkins, 1973). Her conclusion that "semantic and nonsemantic tasks differentially activate the associative meaning of words" (Ghatala, 1981, p. 47) is in very close accord with the conclusions drawn by Jenkins and his associates (e.g., Jenkins, 1974). However, her emphasis on the structural characteristics of the stimulus materials seems to go beyond the orienting task literature.

The effects of imagery, of course, may be viewed separately from the role of imagery in orienting tasks. From this perspective, a question about the nature of the to-be-learned materials remains. That is, is the effectiveness of imagery as a memory aid dependent on meaningfulness in the to-be-learned information? The orienting task literature would suggest that this would be the case, but D'Agostino, O'Neill, and Paivio (1977), in a study investigating memory for stimulus material varying in concreteness, employed a dual-coding hypothesis to account for the finding that both semantic and acoustic tasks resulted in greater levels of recall than did a structural task. The dual-coding hypothesis (Paivio, 1975) has been employed to account for the effects of imagery separately from the effects of imagery in orienting tasks. From this vantage point, a question about the nature of the to-be-learned materials remains. That is , is the effectiveness of imagery as a memory aid dependent on the meaningfulness of the to-be-remembered information? The dual-coding hypothesis would suggest that, in the absence of meaningful stimulus materials, imagery should still result in heightened levels of recall. On the other hand, it seems that Jenkins and his associates (e.g., Jenkins, 1974) would suggest that both what the subject does with the stimulus material and the meaningfulness of that material to the subject determine the memorability of the information. Hence, memory aids such as imagery should not be effective in enhancing the memorability of nonmeaningful materials.

A second question involves the relative effectiveness of visual and acoustic imagery. The literature documenting the facilitative effects of visual imagery is quite large (e.g., Paivio, 1975; Yuille \& Paivio, 1968), and the superiority of visual to acoustic encoding has been well established (e.g., Bousfield, Esterson, \& Whitmarch, 1957). Hence, given stimulus items of equal meaningfulness and concreteness, it would seem that visual imagery should be superior to acoustic imagery in facilitating recall and that both should be superior to a control condition not employing imagery.

The current study, which represents an initial investigation, had three major goals: (1) to determine the facilitative effects of imagery in both intentional and incidental learning tasks when the stimulus items are nonmeaningful, (2) to determine the relative effects of visual and acoustic imagery, and (3) to investigate the effects of individual differences in imagery ability.

In Experiment 1, CVCs from Glaze's (1928) list of 101 " 0 " association value CVCs were selected for use based on " 0 " ratings of meaningfulness and concreteness by a pilot group. Recall of CVCs was compared across 
groups asked to memorize the CVCs without further instruction, a group instructed to employ visual imagery, and a group instructed to employ acoustic imagery. Experiment 2 replicated Experiment 1 with the addition of the administration of the Bett's QMI Vividness of Imagery Scale, the results of which were employed as a blocking variable in the data analysis to determine the effects of individual differences in visual imagery ability. Experiment 3 replicated Experiment 2 in an incidental learning paradigm.

\section{EXPERIMENT 1}

\section{Method}

Subjects and Setting. Subjects were 47 high school junior and senior volunteers (ages 16-18 years) who received course credit for participation. All data were collected by one of the experimenters in a classroom set aside for that purpose.

Apparatus. A TRS-80 microcomputer was employed to present the to-be-learned materials to subjects via a 19-in. Sony television monitor.

Materials. The 101 CVCs Glaze (1928) rated at a 0 association value were selected as the stimulus item pool. These CVCs were then rated on two dimensions, meaningfulness and concreteness-abstractness, by a group of 30 high school students drawn from a neighboring school. Thirty-one of the CVCs were rated at 0 on both meaningfulness and concreteness by all the subjects in the pilot group and were selected for use in the current study.

General procedure. Students were randomly assigned to three groups, control (no directions other than to memorize the list), auditory (directions to auditorily imagine the CVCs; i.e., to imagine their sounds), and visual (directions to visually imagine the picture presented by the CVCs). Students in all conditions were told that they would be tested on the materials after presentation. In each presentation of the materials, the CVCs were randomly ordered. Each of 10 CVCs appeared on the screen for $2 \mathrm{sec}$, followed by an 8-sec blank screen prior to the appearance of the next CVC.

Following a 15-sec verbal distraction task, subjects were asked to list all the CVCs they could remember. Five minutes were allowed for this task.

\section{Results}

Students' protocols were scored for the number of correct CVCs recalled. No credit was awarded for partially correct responses. A simple one-way analysis of variance indicated no significant difference between groups $[F(2,44)=2.384, p>.10]$. Means and standard deviations appear in Table 1.

\section{Discussion}

The results of Experiment 1 were generally consistent with the work of Jenkins and his associates (e.g., Jenkins, 1974). That is, imagery was no more effective than were simple directions to memorize when nonmeaningful materials were employed as the

Table 1

Means and Standard Deviations of CVCs Recalled: Experiment 1

\begin{tabular}{lccl}
\hline \multicolumn{1}{c}{ Group } & Mean & SD & N \\
\hline Visual Imagery & 4.07 & 2.21 & 13 \\
Auditory Imagery & 2.94 & 1.52 & 17 \\
Control & 4.06 & 1.22 & 15 \\
\hline
\end{tabular}

Table 2

Means and Standard Deviations of CVCs Recalled: Experiment 2

\begin{tabular}{|c|c|c|c|c|c|c|}
\hline \multirow[b]{3}{*}{ Group } & \multicolumn{6}{|c|}{ Visual Imagery Ability } \\
\hline & \multicolumn{3}{|c|}{ High } & \multicolumn{3}{|c|}{ Low } \\
\hline & Mean & SD & $\mathbf{N}$ & Mean & SD & $\mathrm{N}$ \\
\hline Visual Imagery & 8.10 & 1.46 & 13 & 3.13 & 1.40 & 14 \\
\hline Auditory Imagery & 5.95 & 1.42 & 13 & 2.20 & 1.51 & 13 \\
\hline Control & 4.02 & 1.23 & 13 & 4.07 & 1.30 & 13 \\
\hline
\end{tabular}

to-be-remembered information. However, the fairly large variation in individual scores in the visual imagery condition led us to hypothesize that individual differences in ability to employ imagery might be an important variable. Hence, Experiment 2 was conducted to examine the role of individual differences in imagery ability on recall of information.

\section{EXPERIMENT 2}

\section{Method}

Subjects and Setting. Seventy-nine junior and senior high school volunteers (ages 16-18 years) participated for course credit. All data were collected in a regular classroom by one of the experimenters.

Apparatus. The equipment employed was the same as that in Experiment 1.

Materials. The CVCs were those employed in Experiment 1. Additionally, subjects were administered the Bett's QMI Vividness of Imagery Scale (shortened form; Sheehan, 1967).

General procedure. Procedures were identical to Experiment 1, except for the administration of the Bett's scale prior to the experiment.

\section{Results}

Students' protocols were scored as in Experiment 1, and Bett's scale was scored according to standard directions. A two-way analysis of variance employing conditions and high/low visual imagery ability as the independent variables was performed. The means and standard deviations are reported in Table 2. Significant differences were observed by conditions $[F(2,75)=5.55$, $\mathrm{p}<.05]$ and imagery ability $[\mathrm{F}(1,75)=6.02, \mathrm{p}<.05]$. There was, additionally, a significant interaction $[F(2,75)=5.94, p<.05]$. Post hoc comparisons via the Tukey procedure $(\alpha=.05)$ indicated that high visual imagery ability subjects in the visual imagery condition recalled a significantly greater number of CVCs than subjects in any other condition and high visual imagery ability subjects in the auditory imagery condition recalled significantly more CVCs than the subjects in any of the other conditions but significantly less than high imagery subjects in the visual imagery conditions. No other comparisons yielded significant differences.

\section{Discussion}

Our hypothesis concerning the effect of individual differences in imagery ability was borne out. Subjects identified as high visual imagers recalled significantly greater numbers of CVCs than other subjects, when given instructions to employ imagery. These findings seem to indicate that the dual-coding theory is appropriate in accounting for the results when individual differences in ability to image are considered. However, because Experiments 1 and 2 involved an intentional learning task 
rather than an incidental task, a third experiment was conducted to verify the results of Experiment 2 with an incidental learning task.

\section{EXPERIMENT 3}

\section{Method}

Subjects and Setting. Ninety undergraduate volunteers (ages 18-22 years) participated for course credit. All data were collected by one of the experimenters in a regular classroom.

Apparatus. A Wollensak 1505 cassette recorder was employed to drive a Kodak Carousel projector that presented the CVCs on a screen.

Materials. The materials were identical to those employed in Experiment 2.

General procedure. The general procedure was adapted from Ghatala's (1981) methods. Subjects were tested in groups of five to eight, with Bett's QMI administered according to standard directions prior to the implementation of experimental procedures. The CVCs were presented on the screen as previously described. Subjects in the experimental conditions were informed that the purpose of the experiment was to obtain ratings of letter groupings for a future experiment (the real purpose of the experiment was explained later). Additionally, all subjects were told that there was a wide variation in the letter combinations they would see and that they would probably be employing most of the points on the scales they were to fill out.

Subjects in the visual imagery condition were asked to judge the ease of visualizing a picture based on each letter sequence (CVC) on a 5-point scale. Subjects in the auditory condition were asked to judge the ease of imaging the sounds of each letter sequence on a 5-point scale. Subjects in the control group were asked to memorize the list of CVCs. Following the presentation of the CVCs, subjects were given a 1-min arithmetic distractor task and then allowed 5 min to recall as many of the CVCs as possible.

\section{Results and Discussion}

Significant main effects were observed by imagery ability $[\mathrm{F}(1,84)=27.61, \mathrm{p}<.01]$ and conditions $[\mathrm{F}(2,84)$ $=4.95, \mathrm{p}<.01]$. The interaction between these variables was also significant $[\mathrm{F}(2,84)=16.77, \mathrm{p}<.01]$. Tukey post hoc comparisons $(\alpha=.05)$ revealed that the high visual imagery subjects in the visual imagery condition were superior to each other condition. Additionally, the high visual imagery ability subjects in the control group were superior to the remaining conditions, whereas the low visual imagery ability subjects in the visual imagery condition recalled significantly fewer CVCs than did subjects in any other condition (see Table 3).

These results generally support the findings of Experi-

Table 3

Means and Standard Deviations of CVCs Recalled: Experiment 3

\begin{tabular}{lcccccc}
\hline & \multicolumn{5}{c}{ Visual Imagery Ability } \\
\cline { 2 - 7 } \multicolumn{1}{c}{ Group } & Mean & SD & N & Mean & SD & N \\
\hline Visual Imagery & 7.13 & 1.68 & 15 & 2.93 & 1.33 & 15 \\
Auditory Imagery & 3.93 & 1.03 & 15 & 3.73 & 1.28 & 15 \\
Control & 4.60 & 1.80 & 15 & 4.07 & 1.62 & 15 \\
\hline
\end{tabular}

ment 2 and indicate the influence of individual differences in visual imagery ability on incidental learning tasks.

\section{GENERAL DISCUSSION}

The results of the current series of experiments can be summarized as follows: First, when individual differences in visual imagery ability are not considered as an independent variable, imagery does not appear to enhance recall of nonmeaningful material. Second, when individual differences are considered, imagery is a significant factor in the recall of nonmeaningful information. Third, when visual imagery ability is employed as an independent variable, visual imagery is superior to auditory imagery. Fourth, when visual imagery ability is considered in an incidental learning task, the results support those obtained in an intentional task.

The current results, when visual imagery ability is considered, support the dual-coding theory. That is, visual imagery alone, whether in an intentional or incidental learning task, resulted in a significantly greater amount of recall than control conditions. However, the dual-coding theory is not supported when individual differences are not taken into account. It appears that considerably more work is required (especially in auditory imagery ability) in examining individual differences with respect to the dual-coding theory and its competitors before any firm conclusions can be drawn.

\section{REFERENCES}

Bousfield, W. A., Esterson, J., \& Whitmarch, G. A. The effects of colored and uncolored pictorial representations on the learning of stimulus words. Journal of Applied Psychology, $1957,41,165-168$.

D’Agostino, P. R., O'Neill,L B. J., \& Paivio, A. Memory for pictures and words as a function of level of processing: Depth or dual coding? Memory \& Cognition, 1977, 5, 252-256.

Ghatala, E. S. Imagery and associative processing effects on incidental memory. Journal of Experimental Psychology: Human Learning and Memory, 1981, 7, 47-55.

Glaze, J. A. The association value of nonsense syllables. Journal of Genetic Psychology, 1928, 35, 255-267.

HYDE, T. S., \& JENkins, J. J. Differential effects of incidental tasks on the organization of recall of a list of highly associated words. Journal of Experimental Psychology, 1969, 82, 472-481.

Hyde, T. S., \& Jenkins, J. J. Recall for words as a function of semantic, graphic, and syntactic orienting task. Journal of Verbal Learning and Verbal Behavior, 1973, 2, 471-480.

Jenkins, J. J. Remember that old theory of memory? Well, forget it! American Psychologist, 1974, 29, 785-795.

Paivio, A. Boding distinctions and repetition effects in memory. In G. H. Bower (Ed.), The psychology of learning and motivation (Vol. 9). New York: Academic Press, 1975.

Shemhan, P. W. A shortened form of Bett's questionnaire upon mental imagery. Journal of Clinical Psychology, 1967, 3, 686-689.

Walsh, D. A., \& Jenkins, J. J. Effects of orienting tasks on free recall in incidental learning: "Difficulty," "effort," and "process" explanations. Journal of Verbal Learning and Verbal Behavior, 1973, 12, 481-488.

Yuille, J. G., \& PAIvio, A. Imagery and verbal mediation instructions in paired associate learning. Journal of Experimental Psychology, 1968, 78, 436-441.

(Received for publication June 23, 1981.) 